\title{
What Can We Learn From The Brain? Functional Brain Mechanisms for Computer-Aided Diagnosis
}

\author{
Bart M. ter Haar Romeny \\ Eindhoven University of Technology \\ Department of Biomedical Engineering \\ The Netherlands
}

\begin{abstract}
Discoveries on brain mechanisms have really taken off. We see spectacular developments in deep learning and convolutional neural network applications, the mathematical understanding of functional circuits, and the availability of massively parallel computing power. Modern optical and new MRI technologies give insight in this spectacular organ, especially in the field of vision. The lecture will address a number of lessons to learn from the brain for medical computeraided diagnosis, explain the mathematical intuition of a range of brain-inspired algorithms, and show some remarkable successes in the field of brain connectivity studies and retinal image analysis.
\end{abstract}

